\section{Monitoring Wound Healing of Diabetic Foot Ulcers Using Two-Dimensional and Three-Dimensional Wound Measurement Techniques: A Prospective Cohort Study}

\author{
Line Bisgaard Jørgensen, ${ }^{1-3, *}$ Ulrich Halekoh, ${ }^{4}$ Gregor B.E. Jemec, ${ }^{5}$ \\ Jens Ahm Sørensen, ${ }^{6}$ and Knud Bonnet Yderstræde ${ }^{1,3}$ \\ ${ }^{1}$ Steno Diabetes Center Odense and ${ }^{6}$ Department of Plastic Surgery, Odense University Hospital and Faculty of Health \\ Sciences, University of Southern Denmark, Odense, Denmark. \\ ${ }^{2}$ OPEN, Odense Patient Data Explorative Network; ${ }^{3} \mathrm{CIMT}$, Centre for Innovative Medical Technology; Odense University \\ Hospital, Odense, Denmark. \\ ${ }^{4}$ Department of Epidemiology, Biostatistics and Biodemography, University of Southern Denmark, Odense, Denmark. \\ ${ }^{5}$ Department of Dermatology, Zealand University Hospital, Roskilde and Faculty of Health Sciences, University of \\ Copenhagen, Copenhagen, Denmark.
}

Objective: Three-dimensional (3D) wound measurements enable the evaluation of wound healing from the wound bed, which may be a more sensitive measure than traditional two-dimensional (2D) area measurements at the level of the skin surface. Few studies have investigated 3D measurements for monitoring wound healing over time. The aim of this study was to compare the changes in 3D area measurements with traditional (2D) area measurements of diabetic foot ulcers during 8 weeks.

Approach: In a prospective cohort study, we monitored the wound healing of 150 diabetic foot ulcers using the 3D-wound assessment monitor camera (3D area) and the digital imaging method (2D area) at baseline, weeks 2,4 , and 8 . The following statistical approaches were used to compare the changes in $2 \mathrm{D}$ area and 3D area measurements: linear regression analysis, paired Wilcoxon rank test, Bonett-Price 95\% confidence interval, and Spearman's correlation coefficient.

Results: The overall changes in $3 \mathrm{D}$ area were significantly larger compared with $2 \mathrm{D}$ area as assessed by Wilcoxon test $(p<0.0001)$ and Bonett-Price 95\% confidence interval of 0.009-0.163. In addition, comparison of $2 \mathrm{D}$ area and 3D area measurements showed that the majority of the wounds had larger decrease in 3D area compared with $2 \mathrm{D}$ area over time.

Innovation: 3D area appears to provide a more sensitive method to monitor wound healing.

Conclusion: Our study highlights the potential benefit of a novel 3D camera for monitoring wound healing of diabetic foot ulcers, which was found particularly useful in monitoring wound healing in large wounds. However, in small wounds $\left(<5 \mathrm{~cm}^{2}\right)$, the $2 \mathrm{D}$ area and $3 \mathrm{D}$ area measurements perform equally well in monitoring the wound healing.

Keywords: diabetic foot ulcers, lower extremity ulcers, wound healing, monitoring, three-dimensional wound measurements, imaging

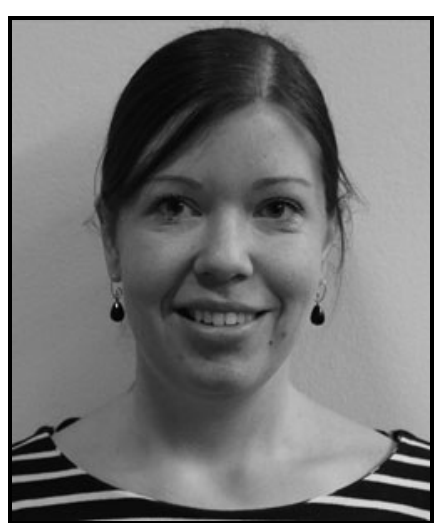

Line Bisgaard Jørgensen, MD

Submitted for publication March 23, 2019. Accepted in revised form June 30, 2019.

${ }^{*}$ Correspondence: Steno Diabetes Center Odense (SDCO), Odense University Hospital and Faculty of Health Sciences, University of Southern Denmark, Sdr. Boulevard 29, Odense C 5000, Denmark

(e-mail: line.bisgaard.joergensen@rsyd.dk) 


\section{INTRODUCTION}

DIABETIC FOOT ULCERS constitute a major complication of diabetes mellitus. It occurs in 19-34\% of diabetic patients and has a 1-year recurrence rate of $40 \% .^{1}$ The diabetic foot is associated with substantial morbidity and mortality, and remains a major risk factor for lower leg amputation. ${ }^{2}$ In addition to individual morbidity, the management of diabetic foot ulcers constitutes a significant economic burden on the health care system. ${ }^{3,4}$

Wound measurement is ideally applied for monitoring the wound healing process, including assessments of treatment effect in routine clinical practice and more explicitly in clinical trials. ${ }^{5}$ When monitoring wound healing, it is essential to use an accurate wound measurement method, which is able to detect small changes in the wound size, and thereby inform and guide treatment strategies. ${ }^{6}$

Accurate wound measurement constitutes a practical problem. ${ }^{5}$ Clinical practice has so far relied almost exclusively on two-dimensional (2D) wound measurement techniques, although these are not able to evaluate wound depth, thereby potentially missing an important aspect of wound healing. In addition, the traditional wound measurements do not take the following into account: undermined wounds, identification of the wound margin, change of patient position affecting the appearance of the wound, and the natural curvature of the body parts (e.g., heel, toe, lower leg). ${ }^{7,8}$

In recent years, several three-dimensional (3D) wound measurement devices have been developed to overcome this. These include laser scanners, structured light techniques, digital imaging, and stereophotogrammetry (SPG) systems. ${ }^{9-12}$ The 3D devices are able to evaluate wound healing from a volume perspective but not commonly used in clinical practice due to several practical limitations, including inaccuracy, high costs, time consumption, and complexity in handling. ${ }^{5,6}$ Few of these systems have become commercially available, including the Eykona ${ }^{\circledR}$ (Fuel 3D, Los Angeles), Silhouette ${ }^{\circledR}$ camera (Aranz, Christchurch, New Zealand), InSight ${ }^{\circledR}$ (eKare, Inc., Fairfax, VA), and LifeViz ${ }^{\circledR}$ 3D system (Quantificare S.A., Valbonne, France). ${ }^{13}$ A recent study compared two of these cameras, the Eykona and Silhouette camera, with digital planimetric method (area) and water displacement (volume) using artificial wound models. ${ }^{10}$ The study showed that the area measurements were relatively close to each other with few percentage deviations using the three methods. However, the Eykona and Silhouette camera overestimated the wound volume with $58 \%$ and 23\%, respectively. ${ }^{10}$ Davis et $a l .{ }^{9}$ evaluated the
LifeViz 3D system, finding no significant difference between expert and nonexpert rater measurements for surface area, depth, and volume. However, the $3 \mathrm{D}$ system was limited by not being able to measure wounds with diameter $>6 \mathrm{~cm}$.

\section{CLINICAL PROBLEM ADDRESSED}

Besides being accurate for measuring wound size, the wound measurement methods should be useful for monitoring wound healing over time. Only few studies with a limited number of study participants or wound models have investigated $3 \mathrm{D}$ wound measurement over time leaving the concept of volume as a superior marker of wound healing largely untested. , $^{9,11,14}$

In this context, we have developed a 3D-wound assessment monitor (WAM) camera, which is able to measure wound size in three dimensions and to assess wound characteristics. We have recently validated the $3 \mathrm{D}$ wound measurements, and found higher intrarater and inter-rater reliability as reflected by high intraclass and interclass correlation coefficients and more narrow 95\% prediction intervals for the $3 \mathrm{D}$ area measurements compared with $2 \mathrm{D}$ area measurements. ${ }^{6}$

The aim of this study was to compare wound healing rates of diabetic foot ulcers using the $3 \mathrm{D}$ area measurements (3D-WAM camera) with those using traditional $2 \mathrm{D}$ area measurements (digital imaging method).

\section{MATERIALS AND METHODS}

We conducted a prospective cohort study at the multidisciplinary clinic: University Centre for Wound Healing, Odense University Hospital, Denmark from August 2015 to April 2018. Diabetic patients ( $\geq 18$ years of age) referred from a general practice or another hospital department for treatment of a foot ulcer were recruited at their first visit in the multidisciplinary clinic or first visit after surgery, including amputation of a toe or forefoot, and wound revision. Exclusion criteria included wounds with surface area $<0.25 \mathrm{~cm}^{2}$ with no substantial loss of subcutaneous tissue and patients with conditions that could affect the compliance (psychiatric disease, dementia). The patients were followed for 1 year or until healing, amputation, or death.

At baseline, information on patient demographics and wound characteristics was collected. The wounds were measured using the 3D-WAM camera and $2 \mathrm{D}$ digital imaging method. The measurements were repeated after $2,4,8,12,24,36$, and 52 weeks or until healing, amputation, or death. At each visit, the first author performed the wound 
measurements described below after cleansing and debridement of the wounds. During the study, the patients were treated with standard wound care, including debridement, cleansing, wound dressings, and offloading. ${ }^{15}$

The study was carried out under the supervision of the local good clinical practice (GCP) unit at Odense University Hospital in accordance with GCP standards. All the data were collected using the electronic REDCap database. ${ }^{16}$

\section{Wound measurement methods (3D area and 2D area)}

The wounds were measured using two different methods: the 3D-WAM camera (3D area) and the digital imaging method (2D area). ${ }^{6,17}$

The 3D-WAM camera is a handheld device, which is developed by the Danish company Teccluster. ${ }^{6}$ The 3D camera comprises a projector and three cameras combined in one device. All the wound measurements were performed in a standardized approach. Using this setup, three photographs are taken from different angles of the wound (one photograph parallel to the wound) within the same distance guided by two red spots pointing at the wound from the camera (Fig. 1). ${ }^{6}$ Afterward the photographs are combined to one 3D image on a computer monitor by marking equivalent points in the photographs. Subsequently, the margin of the wound is manually traced on the monitor, and the software of the 3D-WAM camera immediately afterward estimates the wound size. From each 3D

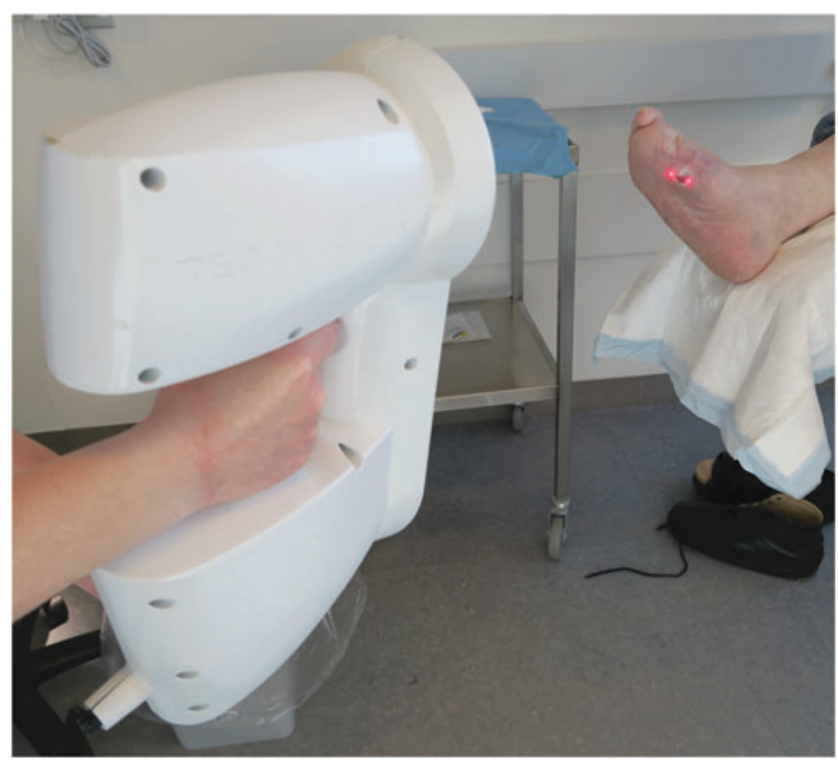

Figure 1. The 3D-WAM camera in use capturing a $3 \mathrm{D}$ image of a diabetic foot ulcer. 3D-WAM, three-dimensional wound assessment monitor. image, four different wound measurements can be obtained: 2D area, 3D area, perimeter, and volume. In this study, we present data on the $3 \mathrm{D}$ area defined as the area of the wound bed, taking into account the curvatures of the wound bed (Fig. 2). ${ }^{6}$

In addition, the digital imaging method is used for measuring the $2 \mathrm{D}$ area defined as the wound surface area. ${ }^{6}$ A photograph of the wound is captured by an iPhone $5 \mathrm{~s}$ and transferred to a computer. Afterward, the software ImageJ (National Institutes of Health, Bethesda, MD) is applied for estimating the $2 \mathrm{D}$ wound area using a ruler parallel to the wound on the photograph. ${ }^{17}$

\section{Approvals}

The study was registered at ClinicalTrials.gov identifier (NCT02423850), and was approved by the Regional Committees on Health Research Ethics for Southern Denmark and the Danish Health and Medicines Authority. All the study participants gave written informed consent.

\section{Statistics}

The aim of the analyses was to compare the changes in the 2D area and 3D area wound measurements over time. In our statistical analyses, we planned to focus on the wound measurements from the first 8 weeks because previous studies have shown that changes in the wound size during the first 4-10 weeks can predict wound healing in diabetic foot ulcers. ${ }^{7,18,19}$ The following statistical approaches were used. First, we estimated the mean changes for each patient in the early time periods through the slopes from simple linear regressions of the 2D area and 3D area measurements on time from baseline to week 8 . The healing rate is defined as the mean changes of wound area

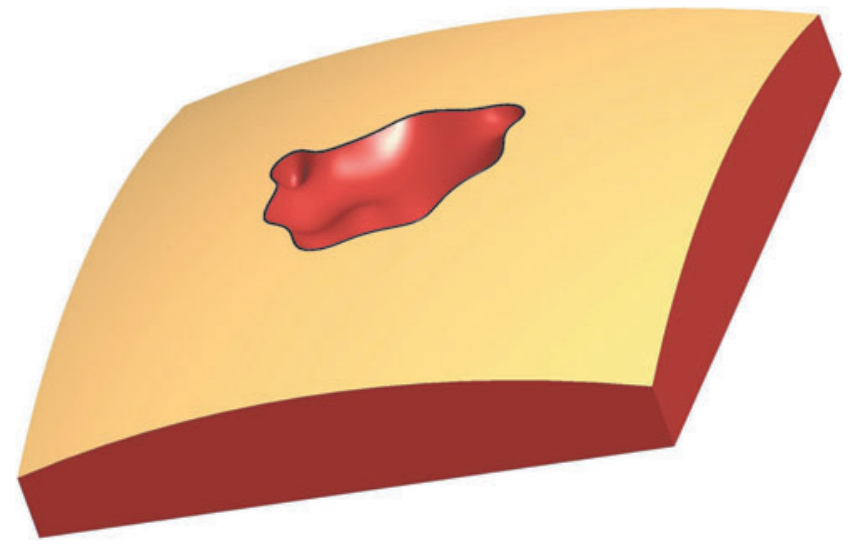

Figure 2. Drawing of a wound to picture the curvatures, which the 3D camera (3D area) is able to measure hereby taking into account the curvatures of the wound bed (red). 
(in $\mathrm{cm}^{2}$ ) per time unit (in weeks), $\frac{\Delta A}{\Delta t}$. Negative values should be interpreted as the wounds are healing (decreasing in size), and positive values should be interpreted as the wounds are nonhealing (increasing in size). The statistical approach was chosen based on the assumption that diabetic foot ulcers are healing in a linear pattern, which has been supported by most previous studies using $2 \mathrm{D}$ wound measurements. ${ }^{7,18,20-22}$ Second, we used the paired Wilcoxon rank test on these slopes to test for a zero median difference between the healing rates of the $3 \mathrm{D}$ area and $2 \mathrm{D}$ area measurements. Furthermore, we provide a confidence interval for the median difference between the healing rates of the $3 \mathrm{D}$ area and $2 \mathrm{D}$ area measurements using the Bonett-Price approach. ${ }^{23}$ The Spearman correlation coefficient was used to quantify the correlations between the changes of $2 \mathrm{D}$ area and $3 \mathrm{D}$ area measurements. Finally, we plotted the difference between the healing rates of $3 \mathrm{D}$ area and $2 \mathrm{D}$ area measurements against the $2 \mathrm{D}$ area measured at baseline. We added a prediction line to the plot using simple linear regression with the Huber-White sandwich estimator to account for heteroskedasticity of the residual variance. ${ }^{24}$ In all the statistical analyses, we excluded the study participants who missed one or more wound measurements during the first 8 weeks.

A sample size of 97 participants was estimated to yield a power of $90 \%$ at a significance level of 0.05 to detect a wound area reduction of $25 \%$ after 4 weeks. ${ }^{25}$ The significance level of the tests was set at a $p$-value of 0.05 . All statistical analyses were carried out using STATA, version $15 .^{26}$

\section{RESULTS}

A total of 382 patients were screened, and 150 patients with diabetic foot ulcers were included in the study (Fig. 3). Of the 150 patients included, 138 were followed for 2 weeks, 130 for 4 weeks, 109 for 8 weeks, 80 for 12 weeks, 38 for 24 weeks, 21 for 36 weeks, and 12 for 52 weeks.

In addition, of the 109 patients who were followed for $\geq 8$ weeks, 13 patients missed one or more wound measurements during the first 8 weeks, leaving 96 patients for statistical analyses. Of the 96 patients, $65(67.7 \%)$ healed within 1 year, 14 (14.6\%) were amputated, 3 (3.1\%) died, 13 (13.5\%) were chronic after 1 year (not healed after 1 year), and $1(1.0 \%)$ was discontinued due to several missing appointments. The mean healing time was 149 days (SD 74) for the healing wounds. Table 1 shows the characteristics of the patients included in the study.

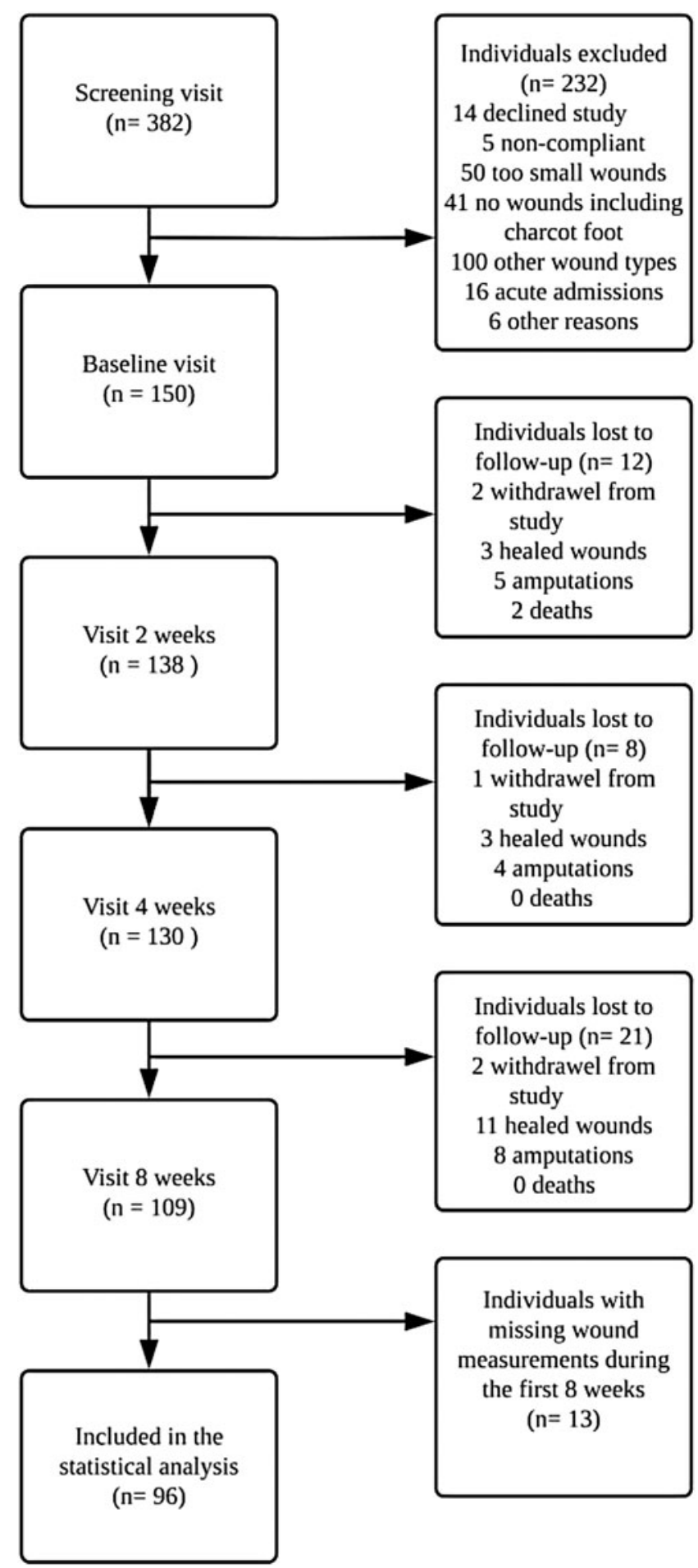

Figure 3. Flow chart of the study participants in the prospective cohort study.

The distribution of the difference between the healing rates of $3 \mathrm{D}$ area and $2 \mathrm{D}$ area measurements was estimated to a median of 0.086 (25-75\% percentile 0.012-0.474). Correspondingly, a paired Wilcoxon rank test for the hypothesis of equality of the two distributions rejects the hypothesis with a 
Table 1. Baseline characteristics of the study participants

\begin{tabular}{lc}
\hline & $\mathrm{n}=150$ \\
\hline Age (years) & $64 \pm 12$ \\
Sex, male/female & $128 / 22$ \\
Diabetes type & \\
$\quad$ Type 1 & 27 \\
$\quad$ Type 2 & 123 \\
Location of wound & \\
Toe & 23 \\
Forefoot & 74 \\
Midfoot & 24 \\
Heel & 21 \\
Ankle & 8 \\
Baseline 2D area $\left(\mathrm{cm}^{2}\right)^{\mathrm{a}}$ & $7.83 \pm 9.17$ \\
Baseline 3D area $\left(\mathrm{cm}^{2}\right)^{\mathrm{a}}$ & $11.94 \pm 14.32$ \\
\hline
\end{tabular}

${ }^{a}$ Values are mean \pm SD.

2D, two dimensional; 3D, three dimensional.

highly significant $p$-value $(p<0.0001)$ and the Bonett-Price $95 \%$ confidence interval of 0.009 , 0.163 for the median difference, which does not contain zero. This signifies that the overall changes in 3D area measurements were larger compared with the changes in 2D area measurements during the 8 weeks period. Finally, there was a significant positive correlation between the changes in $2 \mathrm{D}$ area and $3 \mathrm{D}$ area measurements after 8 weeks assessed by a Spearman correlation coefficient of $0.94(p<0.0001)$, which implies that the $2 \mathrm{D}$ area and $3 \mathrm{D}$ area measurements are changing in the same directions despite the fact that the changes in 3D area measurements were larger compared with the changes in $2 \mathrm{D}$ area measurements.
Figure 4 presents an overview of the healing rates illustrated by $2 \mathrm{D}$ area and 3D area measurements compared with each other during the first 8 weeks. Each point on the figure represents the healing rate for each study participant. The blue dots represent the wounds with larger changes in $3 \mathrm{D}$ areas compared with $2 \mathrm{D}$ areas, and the red triangles represent the wounds with larger changes in $2 \mathrm{D}$ areas compared with $3 \mathrm{D}$ areas. The points closest to the red line illustrate wounds with healing rates that deviate to the least extent from each other using $2 \mathrm{D}$ area versus $3 \mathrm{D}$ area measurements. It is apparent from the figure that $3 \mathrm{D}$ area measurements reveal higher healing rates compared with the 2D area measurements (below the red line) demonstrated by primarily blue dots. Furthermore, a cluster of study participants with healing rates around zero, using both $2 \mathrm{D}$ area and $3 \mathrm{D}$ area measurements, is identified. This demonstrates the wounds that barely changed during the 8 weeks. The study participants with positive healing rates represent the nonhealing wounds. Overall, this implies that the healing rates are considerably larger using 3D area measurements compared with $2 \mathrm{D}$ area measurements in the healing wounds. However, in the nonhealing wounds that were unchanged or increased in size, the healing rates appear almost the same using $2 \mathrm{D}$ area and $3 \mathrm{D}$ area measurements (close to the red line).

Figure 5 illustrates the difference between the healing rates of the $3 \mathrm{D}$ area and $2 \mathrm{D}$ area measurements in relation to the $2 \mathrm{D}$ area measured at

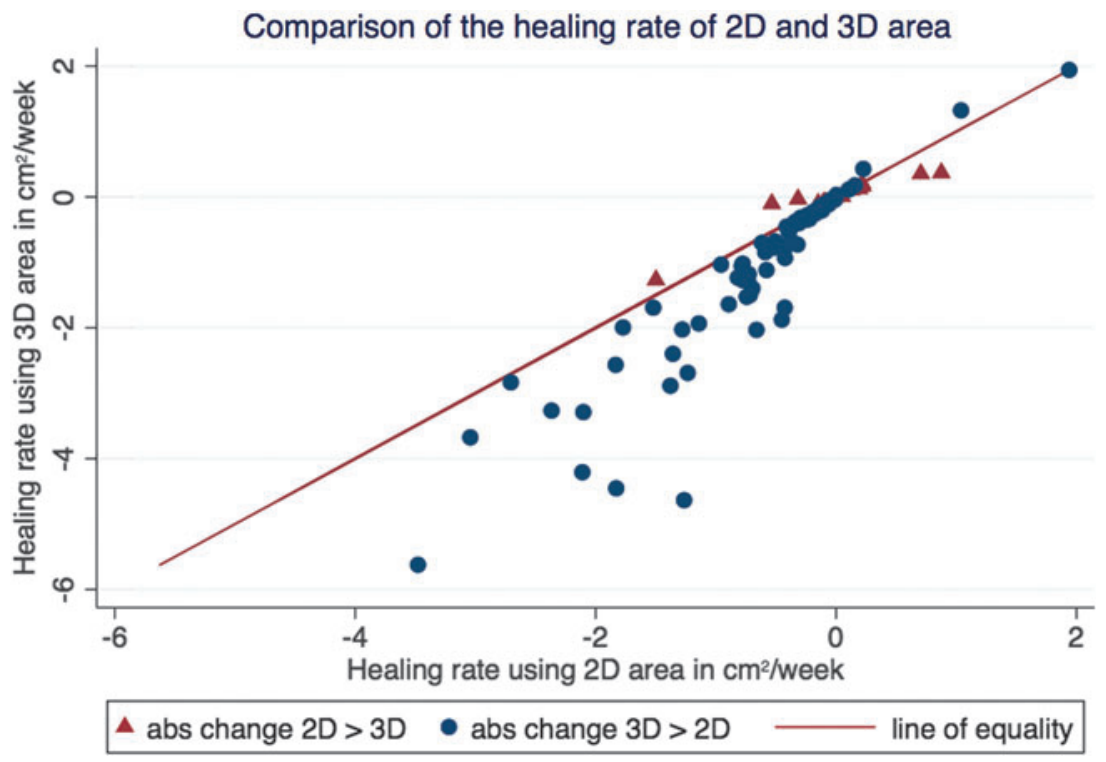

Figure 4. Comparison of the healing rate (slope) of 2D area (digital imaging method) and 3D area (3D-WAM camera) measurements during the first 8 weeks. The $x$-axis represents the healing rate in $\mathrm{cm}^{2} /$ week of $2 \mathrm{D}$ area measurements and the $y$-axis represents the healing rate in $\mathrm{cm}^{2} /$ week of $3 D$ area measurements. The red line illustrates equality between the healing rates of $2 \mathrm{D}$ area and $3 \mathrm{D}$ area measurements, respectively. 2D, two dimensional. 


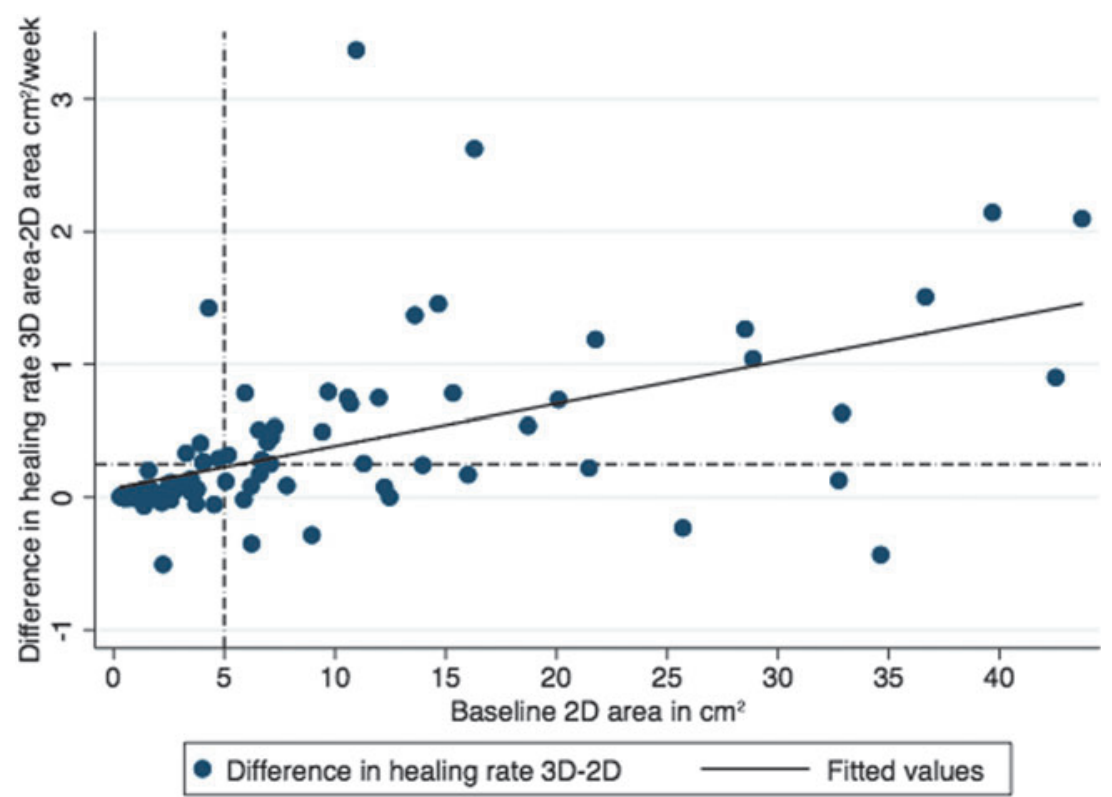

Figure 5. The relationship of the difference between the healing rates of the $3 \mathrm{D}$ area and $2 \mathrm{D}$ area measurements and the $2 \mathrm{D}$ area measured at baseline.

baseline. As shown in the figure, the difference between the healing rates is increasing proportionally although scattered to the $2 \mathrm{D}$ wound area measured at baseline illustrated by the fitted line. We considered a difference of $0.25 \mathrm{~cm}^{2} /$ week between the healing rates of $3 \mathrm{D}$ area and $2 \mathrm{D}$ area measurements as clinically meaningful. We performed an additional analysis in which we estimated the proportion of wounds with baseline 2D area $<5 \mathrm{~cm}^{2}$ and with a difference in healing rate $>0.25 \mathrm{~cm}^{2} /$ week, which amounted to $11.8 \%$, and also the proportion of wounds with baseline $2 \mathrm{D}$ area $>5 \mathrm{~cm}^{2}$ and with a difference in healing rate $>0.25 \mathrm{~cm}^{2} /$ week, which amounted to $66.7 \%$. Therefore, we regard the $3 \mathrm{D}$ area measurements primarily useful for monitoring healing in diabetic foot ulcers with surface area $>5 \mathrm{~cm}^{2}$.

Figures 6-8 represent descriptive plots showing the $2 \mathrm{D}$ area and $3 \mathrm{D}$ area measurements at baseline, weeks 2,4 , and 8 for all the study participants. We divided the study participants into three groups by the difference between the healing rates of 3D area and 2D area measurements: $\geq 0.25 \mathrm{~cm}^{2} /$ week (Fig. 6), $\geq 0-0.25 \mathrm{~cm}^{2} /$ week (Fig. 7), and $<0 \mathrm{~cm}^{2}$ /week (Fig. 8). Figure 6 shows the healing wounds with the largest difference in healing rates of $3 \mathrm{D}$ area and $2 \mathrm{D}$ area measurements. Interestingly, the 3D area and 2D area measurements of the wounds are primarily differing at baseline, and are approaching each other during the 8 weeks period. This would be explained by the fact that the wounds approximate the level of the skin surface, thereby diminishing the difference between the two measurement methods. Furthermore, it can be seen from the figure that most of the wounds are large at baseline. Figure 7 shows the wounds with only minor differences between the healing rates of $3 \mathrm{D}$ area and $2 \mathrm{D}$ area measurements. As seen from the figure, most of the wounds are small, and the changes in $2 \mathrm{D}$ and the $3 \mathrm{D}$ area measurements are small. Few exceptions can be seen from the figure because the wounds are both increasing and decreasing in size, and thereby reducing the overall changes during the study period. Finally, Fig. 8 shows few wounds with slightly higher healing rates using $2 \mathrm{D}$ area compared with $3 \mathrm{D}$ area measurements. The figure depicts either wounds in steady state or wounds that are increasing in size.

\section{DISCUSSION}

In this study, we compared the changes in $3 \mathrm{D}$ area (3D-WAM camera) and $2 \mathrm{D}$ area (digital imaging method) measurements of diabetic foot ulcers during the first 8 weeks of treatment. In accordance with our hypothesis, the study showed that $3 \mathrm{D}$ area measurements detected larger changes compared with $2 \mathrm{D}$ area measurements. Wounds represent area of tissue loss, and during the initial proliferative phase of wound healing, the formation of granulation tissue dominates while epithelialization occurs later. ${ }^{27}$ It is therefore suggested that the reduction in the $3 \mathrm{D}$ wound area may be expected to be a more sensitive indicator of wound 


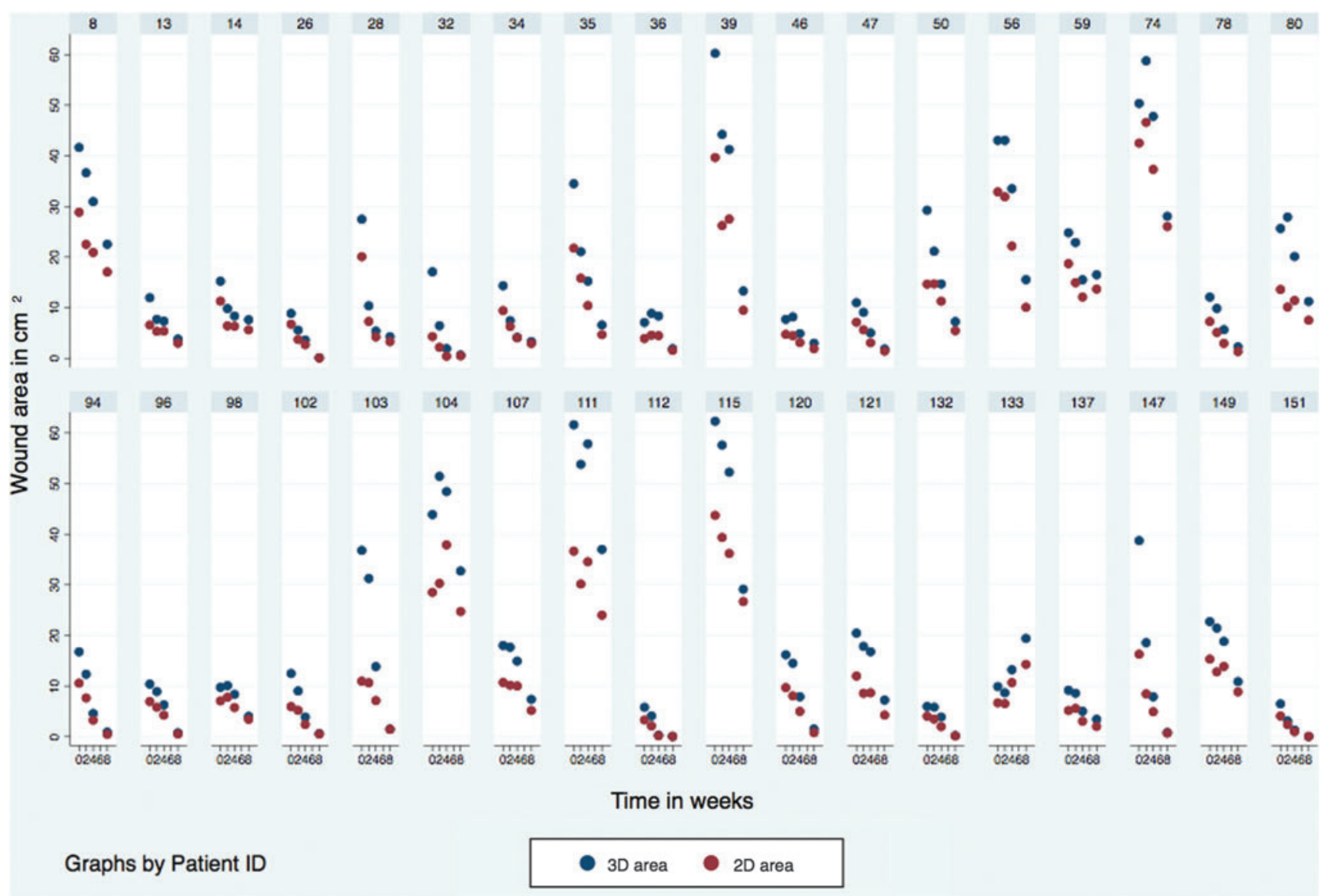

Figure 6. The $2 \mathrm{D}$ area and $3 \mathrm{D}$ area measurements for the study participants depicting the differences between the healing rates of $3 \mathrm{D}$ area and $2 \mathrm{D}$ area measurements $\geq 0.25 \mathrm{~cm}^{2} /$ week.

healing as it is both an expression of the formation of granulation tissue and epithelial tissue, while the reduction in $2 \mathrm{D}$ area (wound surface area) predominantly represents epithelialization. ${ }^{10,28}$ Accordingly, we expected to see a more prominent reduction in the $3 \mathrm{D}$ area compared with $2 \mathrm{D}$ area measurements in the initial phase of the wound healing process. Later in the process, the wounds are superficial, and therefore the $2 \mathrm{D}$ area and $3 \mathrm{D}$ area align more, which is supported by Fig. 6 showing $2 \mathrm{D}$ area and 3D area measurements for the healing wounds. It is therefore speculated that $3 \mathrm{D}$ area measurements of wounds contribute to our understanding of the wound healing process.

Interestingly, our results showed a difference in the healing rates of the $3 \mathrm{D}$ and $2 \mathrm{D}$ areas depending on the wound area measured at baseline. We found that only $11.8 \%$ of the wounds with an area $<5 \mathrm{~cm}^{2}$ at baseline showed a difference $>0.25 \mathrm{~cm}^{2} /$ week between the healing rates of $3 \mathrm{D}$ area and $2 \mathrm{D}$ area during the first 8 weeks. However, most of the wounds $(66.7 \%)$ with baseline area $>5 \mathrm{~cm}^{2}$ had a difference in the healing rates of $3 \mathrm{D}$ area and $2 \mathrm{D}$ area $>0.25 \mathrm{~cm}^{2} /$ week. This may be explained by the fact that most of the wounds with a baseline area $<5 \mathrm{~cm}^{2}$ were superficial, and therefore the difference between the healing rates of $3 \mathrm{D}$ and $2 \mathrm{D}$ areas was small. This implies that the $3 \mathrm{D}-\mathrm{WAM}$ camera seems primarily useful for monitoring healing in diabetic foot ulcers with $2 \mathrm{D}$ area $>5 \mathrm{~cm}^{2}$.

The 2D measurement methods are generally fast, inexpensive, and easy to use. However, the methods also have several limitations, including illumination of the wound, the size and location of the wound (e.g., curved part of the body), and the fact that skewed angles of the camera can lead to underestimation of the $2 \mathrm{D}$ area. ${ }^{5,29-32}$

Recently, various 3D wound measurement methods have therefore been developed, and validated by studies of intrarater and inter-rater reliability or comparison with other measurement methods. ${ }^{5,10,28}$ In contrast, only few studies have investigated the dynamics of 3D measurement methods for monitoring wound healing over time. ${ }^{9,11,14}$ Bills et al. ${ }^{11}$ compared two 3D measurement devices, including the $3 \mathrm{D}$ wound mea- 


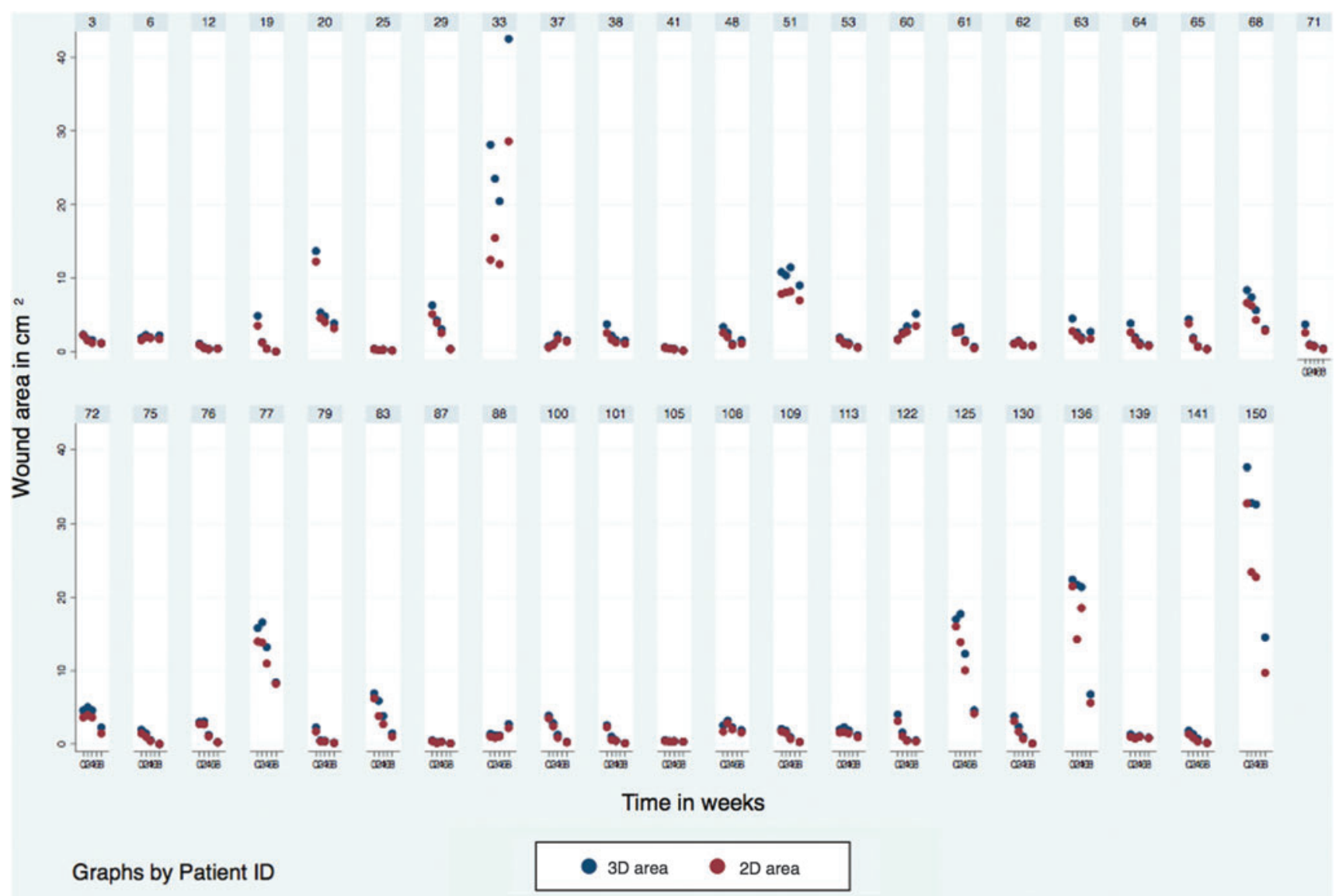

Figure 7. The $2 \mathrm{D}$ area and $3 \mathrm{D}$ area measurements for the study participants depicting the differences between the healing rates of $3 \mathrm{D}$ area and $2 \mathrm{D}$ area measurements between $\geq 0$ and $0.25 \mathrm{~cm}^{2} /$ week.

surement device (InSight ${ }^{\mathrm{TM}}$; eKare, Inc.) and the laser-assisted wound measurement device (Silhouette Star ${ }^{\mathrm{TM}}$; Aranz), by measuring two wound models and simulate wound healing over time using Play-Doh ${ }^{\circledR}$. No statistically significant difference was found between the two devices. Further, two studies have used the same SPG system, 3D LifeViz digital imaging system, for monitoring wound healing. ${ }^{9,14}$ Davis et $a l .{ }^{9}$ compared the wound area using the SPG system and the elliptical method (width $\times$ length $\times \pi$ ) on 13 pressure wounds in a period of 6 weeks, demonstrating significant difference only between width measurements. In addition, Chaby et al. ${ }^{14}$ have compared the changes in area on 44 venous leg ulcers from baseline to 4 weeks using the same SPG system and manual planimetric method. This study showed equivalent changes in the area using either of the two methods. ${ }^{14}$ However, to be widely used in clinical practice, 3D measurement devices should provide additional information about the wound healing process compared with the more simple $2 \mathrm{D}$ measurement methods due to the cost and the time used for training and the everyday use of the equipment. ${ }^{5}$

Compared with the above-mentioned studies, our study is the first to investigate the advantages of using $3 \mathrm{D}$ wound measurements for monitoring wound healing in diabetic foot ulcers compared with the traditional $2 \mathrm{D}$ area measurements in a large cohort. Based on our results, we recommend using 3D wound measurements for monitoring wound healing in large diabetic foot ulcers $>5 \mathrm{~cm}^{2}$. However, in small wounds we found that the 2D and 3D measurement methods perform equally well in monitoring wound healing.

The major strengths of this study include the statistical power of the cohort size (96 individuals with diabetic foot ulcers), the data density (four consecutive measurements during 8 weeks [384 measurements in total]), and the fact that the study followed GCP guidelines and underwent monitoring. Moreover, only one clinician performed the wound measurements using the 3D-WAM camera and digital imaging method, effectively eliminating inter-rater variability. ${ }^{6}$ 


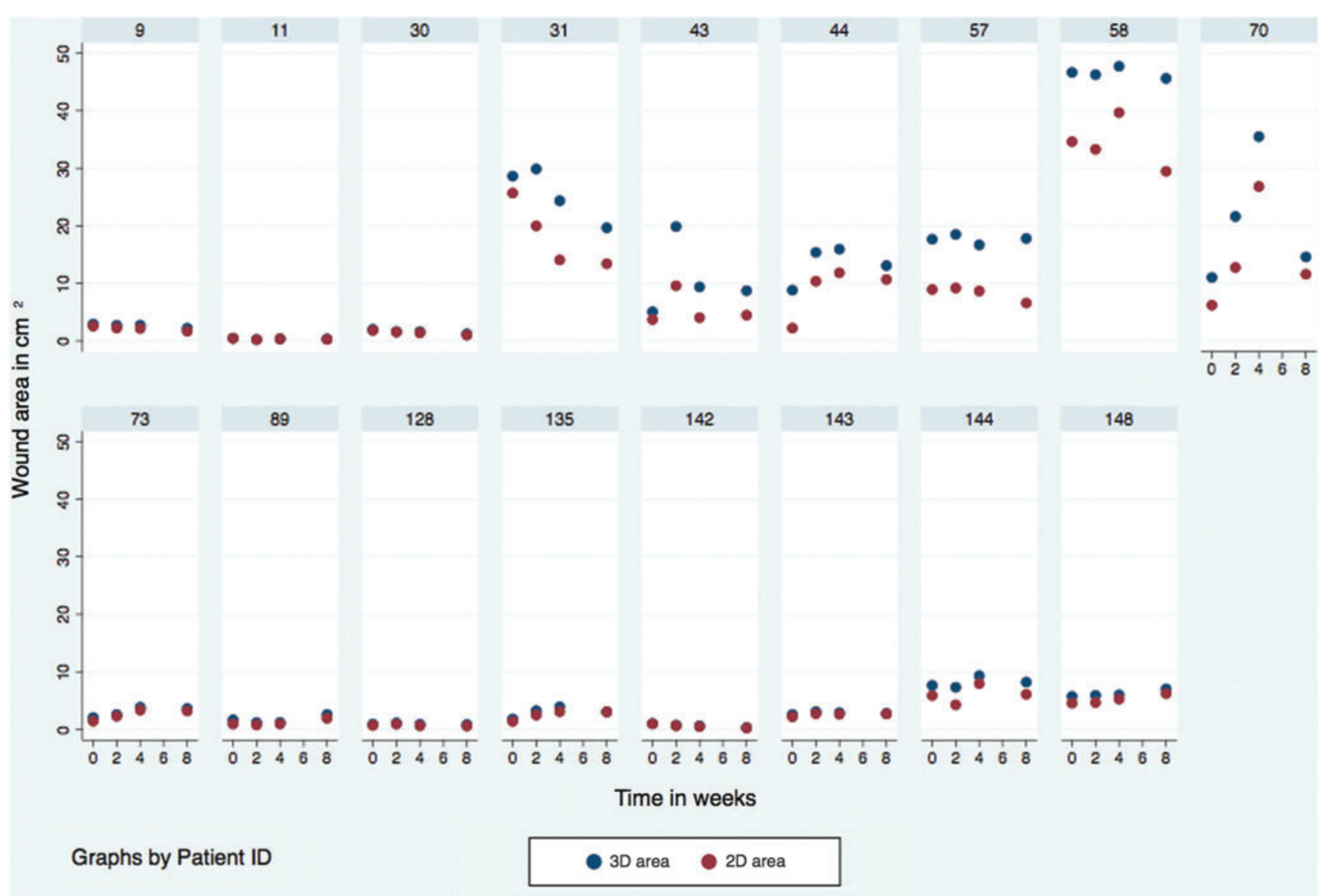

Figure 8. The $2 \mathrm{D}$ area and $3 \mathrm{D}$ area measurements for the study participants depicting the differences between the healing rates of $3 \mathrm{D}$ area and $2 \mathrm{D}$ area measurements $<0 \mathrm{~cm}^{2} /$ week.

The 3D-WAM camera had several strengths, including ease of use, portability, and not being limited by the size of the wound like other 3D systems. ${ }^{9,28}$ Furthermore, it was easier to identify the wound margin compared with the $2 \mathrm{D}$ images due to the extra dimension in the 3D images. Finally, the $3 \mathrm{D}$ area was a measure of the wound bed taking into account the unevenness and curvatures of the wound bed and the body part. ${ }^{6}$

Our study has some limitations: this is an observational study, meaning that the treatment was not standardized. However, the aim of the study was not to compare the treatments, but only to monitor the wound healing using two different methods regardless of the treatment. Wounds were cleaned before imaging, minimizing the risk of wound care material residue or debris technically interfering with imaging.

Furthermore, the generalizability of our results beyond diabetic foot ulcers may be limited. However, diabetic foot ulcers are often complex with different morphologies, including depth, location on the foot, and etiology. Accordingly, the results might still be useful for other morphologically simpler and often larger wound types. Finally, the 3D-WAM camera had some technical limitations (i.e., inability to measure undermined part of the wounds). However, this is a common issue with all wound measurement methods. ${ }^{6}$

\section{CONCLUSION}

Our study highlights the potential benefit of a novel 3D camera for monitoring wound healing in diabetic foot ulcers. Importantly, this study shows that the $3 \mathrm{D}$ area measurements using the $3 \mathrm{D}-\mathrm{WAM}$ camera are particularly useful in monitoring wound healing in large diabetic foot ulcers. In small wounds with an area $<5 \mathrm{~cm}^{2}$, we found that the $2 \mathrm{D}$ area and $3 \mathrm{D}$ area measurements performed equally well.

Further research should be undertaken to determine the potential use of the 3D-WAM camera for estimating the percentage of the wound represented by granulation tissue, which could contribute additional information about the wound healing process dynamics. ${ }^{7,33}$ 


\section{INNOVATION}

A novel handheld 3D-WAM camera is presented, which is able to measure wound size in three dimensions, thereby monitoring wound healing from the wound bed. ${ }^{6}$ In contrast, the traditional $2 \mathrm{D}$ measurement methods are only able to monitor wound healing from the skin surface. ${ }^{5} \mathrm{We}$ compared the changes in $3 \mathrm{D}$ area and $2 \mathrm{D}$ area measurements of diabetic foot ulcers, and found that the $3 \mathrm{D}$ area measurements were more sensitive to change than $2 \mathrm{D}$ area measurements in large diabetic foot ulcers. It is therefore suggested that $3 \mathrm{D}$ area may perform better in detecting small changes in the early wound healing and thus to guide treatment options in wound healing.

\section{ACKNOWLEDGMENTS AND FUNDING SOURCES}

This study was financially supported by Odense University Hospital Free Research Fund and the Bikuben Fund.

\section{AUTHOR DISCLOSURE AND GHOSTWRITING}

No competing financial interests exist. The content of this article was expressly written by the authors listed. No ghostwriters were used to write this article.

\section{ABOUT THE AUTHORS}

Line Bisgaard Jørgensen, MD, is a $\mathrm{PhD}$ student at the University of Southern Denmark, and the current work is part of her $\mathrm{PhD}$ thesis, which has

\section{KEY FINDINGS}

- 3D wound measurements enable the evaluation of wound healing from the wound bed, which may be a more sensitive measure than traditional $2 \mathrm{D}$ area measurements.

- The overall changes in 3D area were significantly larger compared with $2 \mathrm{D}$ area in accordance with our hypothesis.

- The 3D-WAM camera was found particularly useful in monitoring wound healing of diabetic foot ulcers with surface area $>5 \mathrm{~cm}^{2}$.

- In small wounds with an area $<5 \mathrm{~cm}^{2}$, we found that the $2 \mathrm{D}$ area and $3 \mathrm{D}$ area measurements perform equally well in monitoring the wound healing process.

been focusing on three-dimensional wound measurements for monitoring wound healing of diabetic foot ulcers. Ulrich Halekoh, MSc, PhD, is a biostatistician at the University of Southern Denmark, Denmark. Jens Ahm Sørensen, MD, PhD, is a professor and chairman of the research unit at the Department of Plastic Surgery at Odense University Hospital, Denmark. Gregor B.E. Jemec, MD, DMSc, is a professor and chairman of the research unit at the Department of Dermatology at Roskilde Hospital, Denmark. Knud Yderstræde, MD, PhD, is a consultant diabetologist at Steno Diabetes Center Odense, Denmark. His current research is focusing on developing technical solutions for treatment and monitoring of individuals with chronic diseases in their own home. He has been a part of the consortium patient@home, which has developed $>40$ technical solutions to be used for monitoring a variety of data in chronically affected patients. He has received funding from the Danish Research Council, The Market Development Fund, the NovoNordisk Foundation, and several smaller Funds.

\section{REFERENCES}

1. Armstrong DG, Boulton AJM, Bus SA. Diabetic foot ulcers and their recurrence. N Engl J Med 2017;376:2367-2375.

2. Alavi A, Sibbald RG, Mayer D, et al. Diabetic foot ulcers: Part I. Pathophysiology and prevention. J Am Acad Dermatol 2014;70:1.e-18; quiz 9-20.

3. Boulton AJ, Vileikyte L, Ragnarson-Tennvall G, Apelqvist J. The global burden of diabetic foot disease. Lancet 2005;366:1719-1724.
4. Danish Health Authority. Diabetic foot ulcers-a health technology assessment [In Danish]. Health Technology Assessment 2011;1:28.

5. Jorgensen LB, Sorensen JA, Jemec GB, Yderstraede $\mathrm{KB}$. Methods to assess area and volume of woundsa systematic review. Int Wound J 2016;13:540-553.

6. Jorgensen LB, Skov-Jeppesen SM, Halekoh U, et al. Validation of three-dimensional wound measurements using a novel 3D-WAM camera. Wound Repair Regen 2018;26:456-462.
7. Flanagan M. Wound measurement: can it help us to monitor progression to healing? J Wound Care 2003;12:189-194.

8. Plassmann P, Jones TD. MAVIS: a non-invasive instrument to measure area and volume of wounds. Measurement of Area and Volume Instrument System. Med Eng Phys 1998;20:332338.

9. Davis AJ, Nishimura J, Seton J, Goodman BL, Ho $\mathrm{CH}$, Bogie KM. Repeatability and clinical utility in 
stereophotogrammetric measurements of wounds. J Wound Care 2013;22:90-92, 4-7.

10. Williams KJ, Sounderajah V, Dharmarajah B, Thapar A, Davies AH. Simulated wound assessment using digital planimetry versus threedimensional cameras: implications for clinical assessment. Ann Vasc Surg 2017;41:235-240.

11. Bills JD, Berriman SJ, Noble DL, Lavery LA, Davis KE. Pilot study to evaluate a novel threedimensional wound measurement device. Int Wound J 2016;13:1372-1377.

12. Anghel EL, Kumar A, Bigham TE, et al. The reliability of a novel mobile 3-dimensional wound measurement device. Wounds 2016;28:379-386.

13. Rasmussen BS, Froekjaer J, Joergensen LB, Halekoh $U$, Yderstraede KB. Validation of a new imaging device for telemedical ulcer monitoring. Skin Res Technol 2015;21:485-492.

14. Chaby G, Lok C, Thirion JP, Lucien A, Senet P. Three-dimensional digital imaging is as accurate and reliable to measure leg ulcer area as transparent tracing with digital planimetry. J Vasc Surg Venous Lymphat Disord 2017;5:837-843.

15. Schaper NC, Van Netten JJ, Apelqvist J, Lipsky BA, Bakker K. Prevention and management of foot problems in diabetes: a summary guidance for daily practice 2015, based on the IWGDF guidance documents. Diabetes Res Clin Pract 2017;124:84-92.

16. Harris PA, Taylor R, Thielke R, Payne J, Gonzalez N, Conde JG. Research electronic data capture (REDCap) - a metadata-driven methodology and workflow process for providing translational research informatics support. J Biomed Inform 2009; 42:377-381.

17. Aragon-Sanchez J, Quintana-Marrero Y, AragonHernandez C, Hernandez-Herero MJ. ImageJ: A free, easy, and reliable method to measure leg ulcers using digital pictures. Int J Low Extrem Wounds 2017;16:269-273.
18. Sheehan P, Jones P, Caselli A, Giurini JM, Veves A. Percent change in wound area of diabetic foot ulcers over a 4-week period is a robust predictor of complete healing in a 12-week prospective trial. Diabetes Care 2003;26:1879-1882.

19. Oyibo SO, Jude EB, Tarawneh I, et al. The effects of ulcer size and site, patient's age, sex and type and duration of diabetes on the outcome of diabetic foot ulcers. Diabet Med 2001:18:133-138.

20. Warriner RA, Snyder RJ, Cardinal MH. Differentiating diabetic foot ulcers that are unlikely to heal by 12 weeks following achieving $50 \%$ percent area reduction at 4 weeks. Int Wound $\mathrm{J}$ 2011:8:632-637.

21. Zimny S, Schatz H, Pfohl M. The effects of ulcer size on the wound radius reductions and healing times in neuropathic diabetic foot ulcers. Exp Clin Endocrinol Diabetes 2004;112:191-194.

22. Chaby G, Senet P, Ganry O, et al. Prognostic factors associated with healing of venous leg ulcers: a multicentre, prospective, cohort study. $\mathrm{Br}$ J Dermatol 2013;169:1106-1113.

23. Bonett DG, Price RM. Statistical inference for a linear function of medians: confidence intervals, hypothesis testing, and sample size requirements. Psychol Methods 2002;7:370-383.

24. Huber PJ. The behavior of maximum likelihood estimates under nonstandard conditions. Proceedings of the Fifth Berkeley Symposium on Mathematical Statistics and Probability, Volume 1: Statistics, 221-233, University of California Press, Berkeley, CA, 1967. https://projecteuclid .org/euclid.bsmsp/1200512988 (last accessed September 25, 2020).

25. Kirkwood BR, Sterne JAC. Essential Medical Statistics, 2nd ed. Malden, MA: Blackwell Science Ltd., 2003.

26. StataCorp. 2017. Stata Statistical Software: Release 15. College Station, TX: StataCorp LLC.
27. Sorg H, Tilkorn DJ, Hager S, Hauser J, Mirastschijski U. Skin wound healing: an update on the current knowledge and concepts. Eur Surg Res 2017:58:81-94.

28. Kecelj-Leskovec N, Jezersek M, Mozina J, Pavlovic MD, Lunder T. Measurement of venous leg ulcers with a laser-based three-dimensional method: comparison to computer planimetry with photography. Wound Repair Regen 2007;15:767771.

29. Santamaria N, Ogce F, Gorelik A. Healing rate calculation in the diabetic foot ulcer: comparing different methods. Wound Repair Regen 2012;20: 786-789.

30. Gilman T. Wound outcomes: the utility of surface measures. Int J Low Extrem Wounds 2004;3:125132.

31. Wallenstein S, Brem H. Statistical analysis of wound-healing rates for pressure ulcers. Am J Surg 2004;188:73-78.

32. Gorin DR, Cordts PR, LaMorte WW, Manzoian JO. The influence of wound geometry on the measurement of wound healing rates in clinical trials. J Vasc Surg 1996;23:524-528.

33. Valenzuela-Silva CM, Tuero-Iglesias AD, GarciaIglesias $\mathrm{E}$, et al. Granulation response and partial wound closure predict healing in clinical trials on advanced diabetes foot ulcers treated with recombinant human epidermal growth factor. Diabetes Care 2013;36:210-215.

\section{Abbreviations and Acronyms}

$2 \mathrm{D}=$ two dimensional

$3 \mathrm{D}=$ three dimensional

$\mathrm{GCP}=$ good clinical practice

$\mathrm{SD}=$ standard deviation

$\mathrm{SPG}=$ stereophotogrammetry

WAM $=$ wound assessment monitor 